\title{
Comparison of Apical Root Resorption encountered during Maxillary Incisor retraction using Stainless Steel Boot Loop and TMA Boot Loop - A Case Series
}

\author{
Dr Gaurav Gupta,' Dr Raj Kumar Singh,, Dr Ashima Relhan,, ${ }^{3}$ Dr Gurkeerat Singh,, Dr Abhishek Goyal ${ }^{5}$ \\ 1.5Reader, ${ }^{2,3}$ Assistant professor, ${ }^{4}$ Professor, Department of Orthodontics, \\ Sudha Rustagi College of Dental Sciences, Faridabad, India
}

Correspondence: Dr Raj Kumar Singh; Email: rajortho2010@gmail.com

\section{ABSTRACT}

External apical root resorption (EARR) is probably the most common iatrogenic sequel of orthodontic treatment. The present case series focuses on the incisor retraction using frictionless mechanics. Boot loop made up of .019x.025 Stainless steel or TMA archwire were used for maxillary incisor retraction. Retraction with stainless steel boot loop showed greater root resorption as compared to TMA boot loop.

Key words: apical root resorption, stainless steel boot loop, TMA boot loop

\section{INTRODUCTION}

External apical root resorption (EARR) is probably the most common iatrogenic sequel of orthodontic treatment. Orthodontically induced root resorption has been studied repeatedly in the orthodontic literature, however the etiological factors bear no single consensus and is attributed to be multifactorial.' Inflammatory root resorption is a side effect related to the biological tissue response that enables the teeth to be moved during orthodontic treatment. It was first reported in 1914. Histologically root resorption is found in up to $100 \%$ of orthodontically treated teeth but is less obvious in panoramic and intraoral radiographs. Although the severity of orthodontically induced inflammatory root resorption (OIIRR) is mostly clinically insignificant, the literature shows that $1-5 \%$ of orthodontically treated teeth have severe OIIRR, which is defined as resorption exceeding $4 \mathrm{~mm}$ or a third of the original root length. A significant reduction in the root length can cause an unfavorable crown-root ratio of the affected teeth. ${ }^{2-3}$ This has a great clinical significance,

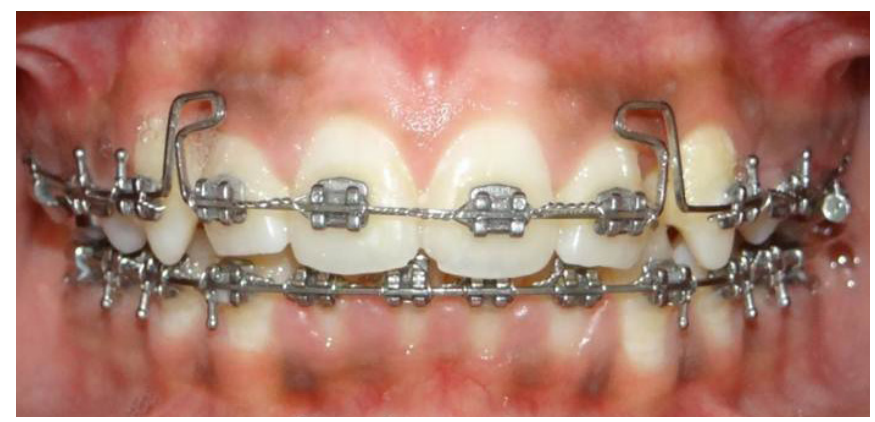

especially when it is coincident with alveolar bone loss or combined with orthodontic retreatment. Apical root loss of 3 $\mathrm{mm}$ is equivalent to $1 \mathrm{~mm}$ of crestal bone loss, which means that periodontal bone loss will reach a critical stage more rapidly if it is accompanied by OIIRR. ${ }^{4}$

The prospective current study included patients having Class II div 1 malocclusion or Class I bimaxillary protrusion cases in the age range of 14-17 years requiring first premolar extraction followed by maximum retraction of incisor teeth. Those cases requiring en-mass retraction of all the anteriors were not included in the study.

The primary purpose of this study was to assess any predisposition of apical root resorption experienced in association with orthodontic treatment mechanics undertaken for space closure using closing loops made of different wire material (Figure 1). A null hypothesis was proposed that there is no difference in the amount of root resorption with both the loops or there is equal amount of resorption.

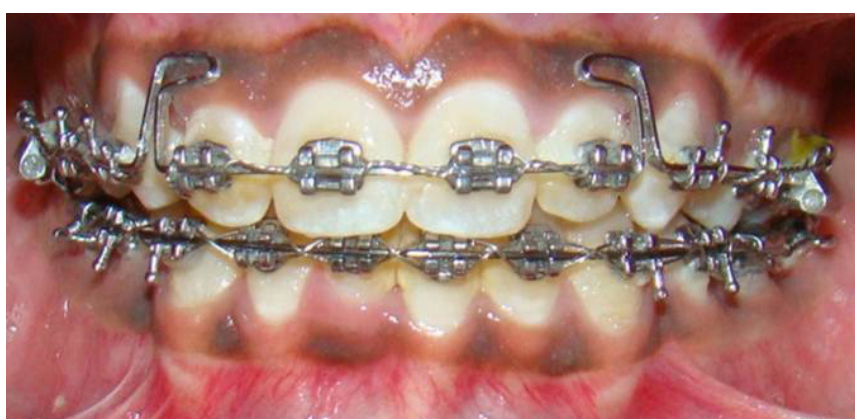

Figure 1: Intraoral photograph of TMA and stainless steel boot loop 
Table 1: Root resorption in patients with stainless steel boot loop

\begin{tabular}{|l|c|l|}
\hline Patient No. & Grade & \multicolumn{1}{c|}{ Inference } \\
\hline 1 & Grade 3 & Accentuated resorption (loss of almost one third of root length) \\
\hline 2 & Grade 2 & Moderate resorption (small area of root loss with the apex exhibiting an almost straight contour) \\
\hline 3 & Grade 2 & Moderate resorption \\
\hline 4 & Grade 2 & Moderate resorption \\
\hline
\end{tabular}

Table 2: Root resorption in patients with TMA boot loop

\begin{tabular}{|l|l|l|}
\hline Patient No. & Grade* & \\
\hline 1 & Grade 0 & Absence of root resorption \\
\hline 2 & Grade 0 & Absence of root resorption \\
\hline 3 & Grade 1 & Mild resorption (root with its normal length and only an irregular contour) \\
\hline 4 & Grade 1 & Mild resorption \\
\hline
\end{tabular}

*Scoring system based on Levander and Malmgren

\section{Case Presentation}

All the cases were first levelled and aligned and canines were retracted till class I canine relation achieved. All the four incisors were consolidated and before putting boot loop $.019 x .025$ stainless steel was left atleast for one month and then boot loop was placed to start incisor retraction. In all cases, space closure was done using frictionless mechanics, either with .019x.025 TMA boot loop or .019x.025 stainless steel boot loop. Average period of retraction in both the groups was around six months. The stainless steel boot loop and TMA boot loop were activated according to their desired force delivery protocols.

The selected cases were subjected to undergo qualitative assessment based on orthopantomogram taken before the placement of boot loop and after the completion of retraction. The pre-retraction and post-retraction orthopantomogram revealed clinically significant external apical root resorption in the group in which retraction was carried out using stainless steel boot loop (Table 1, Figure 6-9) in comparison to TMA boot loop (Table 2, Figure 2-5).


Figure 2: Patient 1: Pre-treatment and post retraction with TMA boot loop
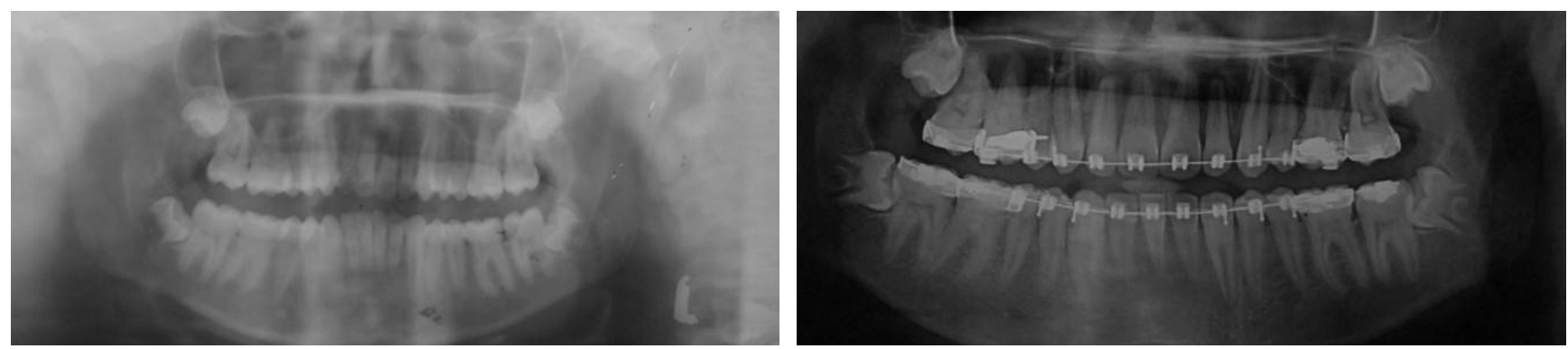

Figure 3: Patient 2: Pre-treatment and post retraction with TMA boot loop 

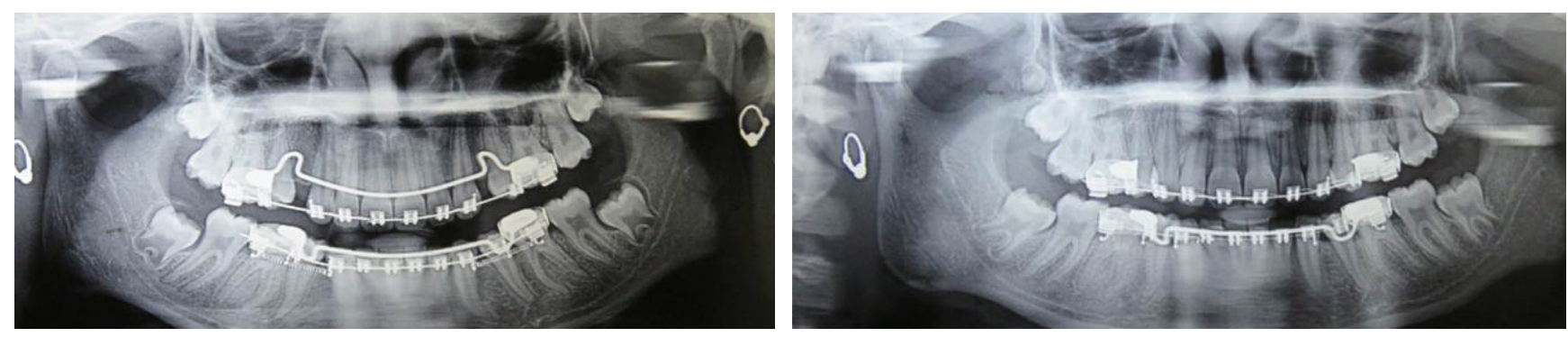

Figure 4: Patient 3: Pre-treatment and post retraction with TMA boot loop

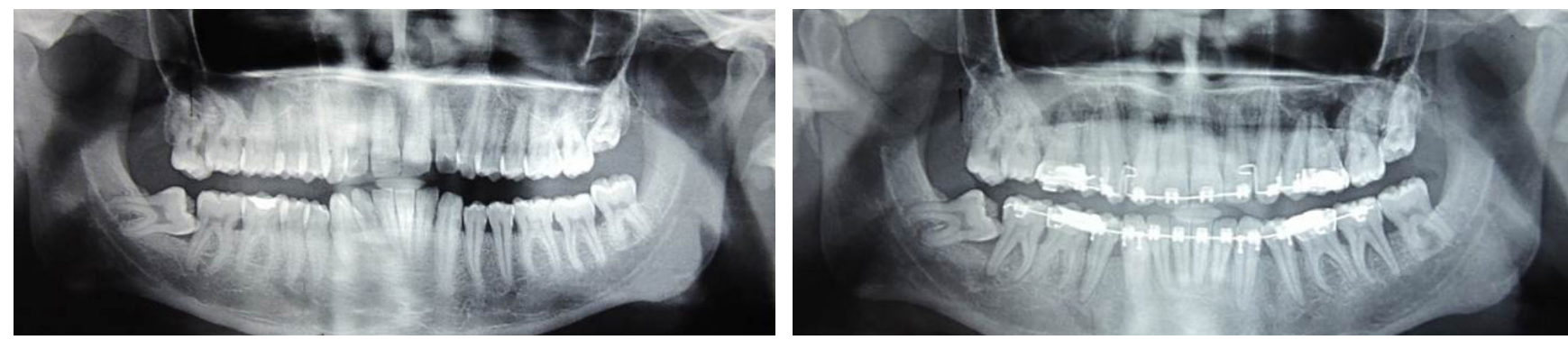

Figure 5: Patient 5: Pre-treatment and post retraction with TMA boot loop
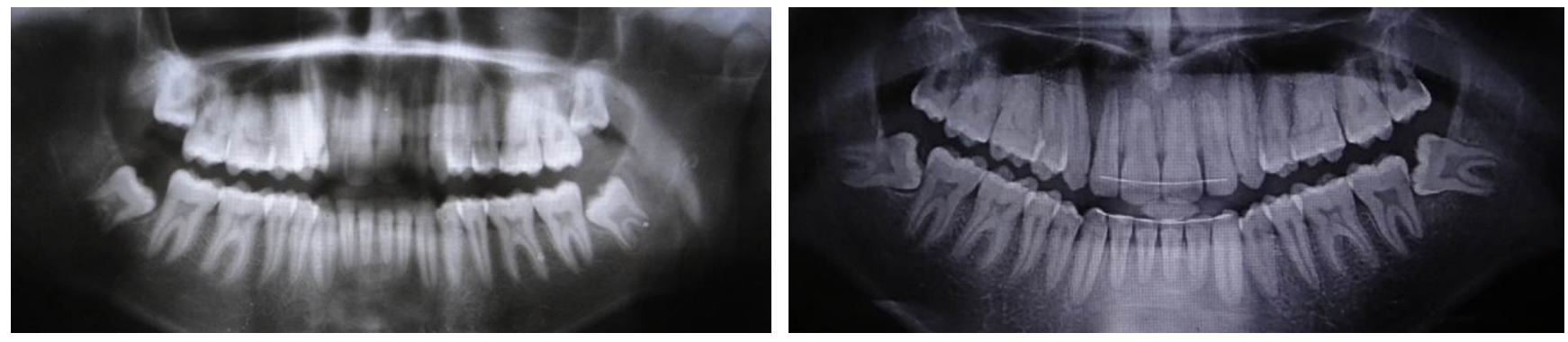

Figure 6: Pre-treatment and post retraction with stainless boot loop

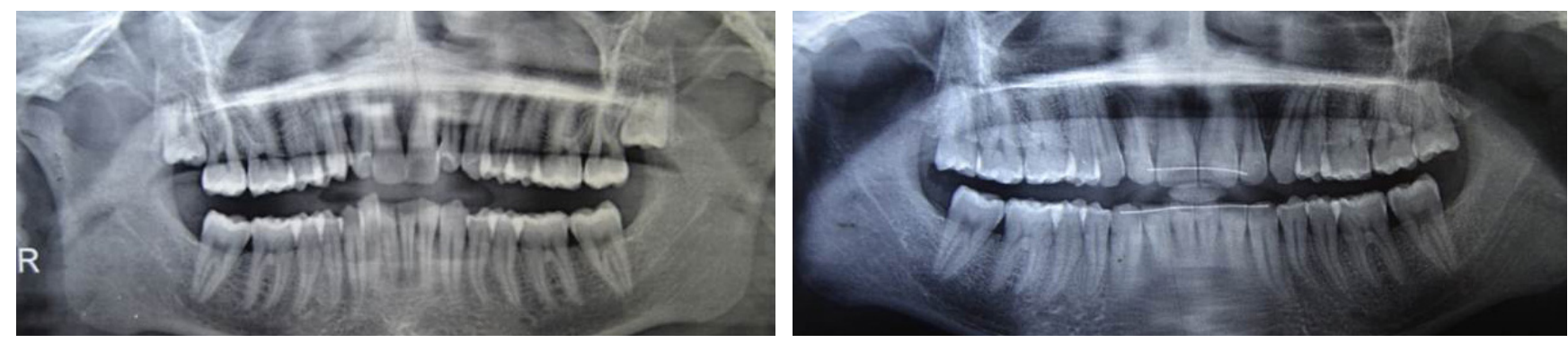

Figure 7: Patient 6: Pre-treatment and post retraction with stainless steel boot loop

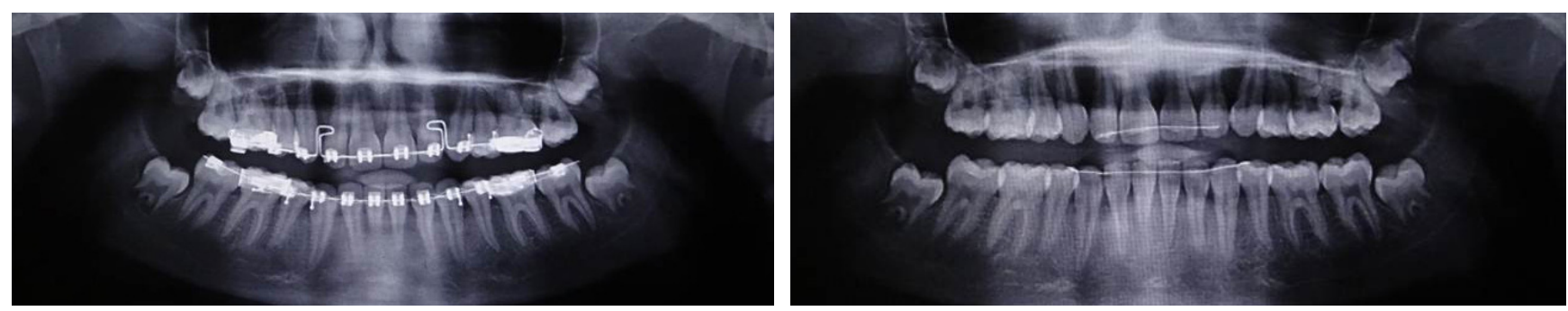

Figure 8: Patient 7: Pre-treatment and post retraction with stainless steel boot loop

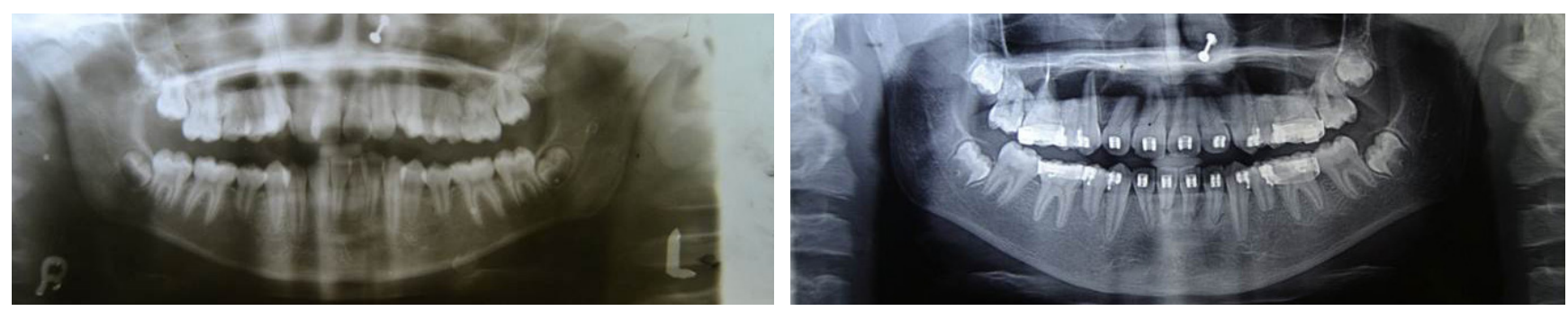

Figure 9: Patient 8: Pre-treatment and post retraction with stainless steel boot loop 


\section{DISCUSSION}

The principal goal that positively permeates the orthodontic practice is the optimal force level and to carry out efficient extraction space closure with minimal undesirable side effects. ${ }^{1-4}$ Apical root resorption is a frequent undesirable side effect during orthodontic treatment. Its multifactorial etiology includes individual predisposition, amount of force applied, characteristics associated to orthodontic treatment, and root morphology. However, root loss resulting from orthodontic treatment does not mostly decrease the longevity or functional capacity of the involved teeth.

Frictional mechanics require more force to bring about tooth movement compared to frictionless mechanics. This force is twice than that is desirable because half is required to overcome friction and the rest is needed to move the tooth. ${ }^{4}$ Extent of root resorption depends upon the amount of force application. Distribution of resorbed lacunae is directly related to the amount of stress on the root surface and the rate of lacunae development is more rapid with increasingly applied forces resulting in more root resorption. ${ }^{5}$ Schwarz found that applied force exceeding the optimal level of 20 $26 \mathrm{gm} / \mathrm{cm}^{2}$ causes periodontal ischemia, which can lead to root resorption. ${ }^{6}$ Comprehensive orthodontic treatment causes increased incidence and severity of root resorption, and heavy forces might be particularly harmful.7-8

The study revealed that the amount of root resorption observed qualitatively on orthopantomogram ${ }^{9}$ was higher in stainless steel boot loop cases (Table 1) as compared to TMA boot loop (Table 2). The extent of observed root resorption was found to be in concordance with the studies which have already been documented in the orthodontic literature..$^{10-13}$
Application of the retraction force during space closure with frictionless mechanics generates a moment on tooth crown that causes an initial crown tipping and later root uprighting. The amount and constancy of force depends on the method of retraction and the material of archwire. A standard vertical loop made in stainless steel wire of .019x.025 dimension delivers a force of around $1000 \mathrm{gm}$ with $1 \mathrm{~mm}$ of activation whereas it is significantly less if fabricated with TMA archwire.

The amount of decay of force is also constant with TMA loop as compared to stainless steel loop. The limitation of stainless steel loop is limited activation as it delivers much higher force with increase in each millimeter of activation whereas TMA exert milder force and requires less frequent activation due to low load deflection rate. In the present study the amount of activation in stainless steel boot loop was less in comparison to TMA boot loop thus keeping in consideration of higher force delivery in stainless steel loop for the same amount of activation.

The present study is a qualitative study and future studies should explore changes in root resorption quantitatively by means of advanced radiodiagnostic aids.

\section{CONCLUSION}

The qualitative assessment of external apical root resorption reveals clinically considerable amount of root resorption in stainless steel boot loop group which can be attributed to higher force delivery by stainless steel loop which accounts to the inherent property of the materials used for retraction.

\section{REFERENCES}

1. Ren Y, Maltha JC, Kuijpers-Jagtman AM. Optimum force magnitude for orthodontic tooth movement: A systematic literature review. Angle Orthod. 2003; 73:86-92.

2. Cattaneo PM, Dalstra M, Melsen B. Strains in periodontal ligament and alveolar bone associated with orthodontic tooth movement analyzed by finite element. Orthod Craniofac Res. 2009; 12:120-8.

3. Zaoui F. Light forces and orthodontic displacement: A critical review. Int J Orthod. 2009; 7:3-13.

4. Van Leeuwen EJ, Kuijpers-Jagtman AM, Von den Hoff JW, Wagener FA, Maltha JC. Rate of orthodontic tooth movement after changing the force magnitude: An experimental study in beagle dogs. Orthod Craniofac Res. 2010; 13:238-45.

5. Harry MR, Sims MR. Root resorption in bicuspid intrusion: A scanning electromicroscopic study. Angle Orthod. 1982; 52:235-58.

6. Schwartz AM. Tissue changes incidental to tooth movement. Int J Orthod. 1932; 18:331-52.

7. Harris EF, Butler ML. Patterns of incisor root resorption before and after orthodontic correction in cases with anterior open bites. Am $J$ Orthod Dentofac Orthop. 1992; 101:112-9

8. Weltman B, Vig K, Fields HW, Shanker S, Kaizar EE. Root resorption associated with orthodontic tooth movement: A systematic review. Am J Orthod Dentofacial Orthop. 2010; 137:462-76.

9. Levander E, Malmgren O. Evaluation of the risk of root resorption during orthodontic treatment: A study of upper incisors. Eur J Orthod. $1988 ; 10: 30-8$.

10. Goldson L, Henrikson CO. Root resorption during Beggs treatment: A longitudinal roentgenologic study. Am J Orthod.1975; 68:55-66.

11. Phillips JR. Apical root resorption under orthodontic therapy. Angle Orthod. 1955; 25:1-22.

12. De Shields RW. A study of root resorption in treated Class II Division 1 malocclusions. Angle Orthod. 1969; 39:231-45.

13. Sharpe W, Reed B, Subtelny JD, Polson A. Orthodontic relapse, apical root resorption, and crestal alveolar bone levels. Am J Orthod Dentofac Orthop. 1987; 91:252-8. 